\title{
Crashworthiness assessment of double-hull tanker structures under ship grounding actions
}

\author{
Aditya Rio Prabowo ${ }^{1, *}$, Jung Min Sohn ${ }^{1,2}$, Dong Myung Bae ${ }^{2}$, and Bangun IR Harsritanto ${ }^{3}$ \\ ${ }^{1}$ Pukyong National University, Interdiscp. Prog. Marine Convergence Design, 48513 Busan, Korea \\ ${ }^{2}$ Pukyong National University, Dept. Naval Architecture and Marine Systems Eng., 48513 Busan, \\ Korea \\ ${ }^{3}$ Universitas Diponegoro, Dept. Architecture, 50275 Semarang, Indonesia
}

\begin{abstract}
Ship grounding is considered as a serious maritime accident which highly contributes to environmental pollution. This impact phenomenon attracts audiences to be studied and assessed since in an instantaneous moment, oil or waste from the damaged bottom tank can spill and contaminate wide water territory. In this study, grounding scenario is modelled as hard grounding where a rock geometry is chosen as representative of oceanic obstruction. The contribution of several main parameters, such as target component and oceanic obstruction are evaluated. The method is to apply a virtual experiment by nonlinear finite element (FE) method to conduct a simulation. A chemical tanker is taken as representative of a marine structure which is subjected to impact load on its bottom structure during interaction with the obstruction. The results indicate that the space between girders provides less resistance in the crushing process. The internal energy in this work is presented to estimate the strength capability of the impacted component, which is followed by the deformation pattern on the lower part of the bottom structure. Finally, the effect of the obstruction on failure sequences in grounding is summarised.
\end{abstract}

\section{Introduction}

There are various forms of maritime accidents which involve ships, offshore structures, sea beds and other entities [1]. A certain accident only produces limited damage on the involved structures, e.g. in the collision case of the Marisa Nusantara in Indonesia [2]. Nevertheless, in other cases, catastrophic chain reactions take place, such as the high profile grounding accident of the Exxon Valdez in Alaska, which has heavily damaged the coastal ecosystem and has become the main cause of species extinction [3]. In the case of ship grounding, the process is very complex and highly nonlinear. Bottom crushing, shell tearing, and steel tension occur when a ship's bottom experiences contact with an obstruction. The fundamental concept to predict structural behaviour under this impact type has been deployed to estimate a mitigation plan, crew evacuation, and safety margin. In order to study these aspects, reference data is a necessity and development for assessment result is required to be steadily performed for wide assumptions.

\footnotetext{
* Corresponding author: aditya.rixx@gmail.com
} 
The present work addresses its focus to the structural response as penetration by an oceanic obstruction is experienced in ship grounding. Certain seabed topology is modelled and augmented by material properties that are possibly encountered in the southern and northern sea routes by a ship. Damage of the bottom structure is presented and the correlation between crashworthiness criteria, such as energy, force, and damage extent are discussed. Finally, a series of structural failure sequences and deformation patterns of the double bottom in grounding are observed.

\section{Impact on marine structures}

\subsection{Pioneer observation}

Maritime transport plays a very important role in international export-import activity. The world has witnessed growth of traffic intensity and massive distribution across the globe using ships. Affected by this trend, society demands rapid development in safety and analysis to ensure that the distribution process by ship can be conducted effectively. Ships have to be robust in facing various loads during its voyage, either static or dynamic load. The second form can occur in accidental situations, such as ship grounding. The indenter may be ship [4] and ice [5] for collision. In grounding, an indenter is defined into several topologies to simplify calculation and analysis [6] and may be denoted as obstruction. Grounding phenomenon is also studied in perspective of risk analysis [7] and the simplified-analytical methodology [8].

\subsection{Internal mechanics: bare plate cut by wedge}

Ship grounding involves various components of a bottom structure with an oceanic obstruction geometry which produce damage on both entities depending on its characteristic (deformable or rigid). A plate cutting is an unavoidable phenomenon in a grounding, which in this case, the bottom plate is to be the main target. Plate cutting can represent structural response and even damage extent of a bottom damage in grounding. Simonsen described some references regarding plate cutting as an obstruction which was represented by a wedge [6]. He classified that plate cutting depended on its deformation mode into:

1. Stable or clean curling cut. In this category, the plate is separated at the tip or in front of the wedge and rolls. After that the torn part folds back to the parallel side in the entire process.

2. Braided cut. The plate separates at the wedge tip as in the stable or clean curling cut. However, the folding does not constantly flaps back on the parallel direction of cutting, but also flaps on the opposite direction (flaps back and forth).

3. Concertina tearing. The target plate folds back and forth in front of the wedge. In this process, the plate is pushed on the cutting direction of the wedge after torn. Space between boundaries on the plate is almost similar with the maximum width of the wedge.

\subsection{Finite elements for impact analysis}

Studies for maritime accidents may be limitless as wide involvements of impact parameters and analysis assumptions make risk and casualty for any grounding phenomenon possibly vary for each scenario. Improvements in methodology and calculation to refine predicted result are sustainably performed. The accuracy of the result is rigorously studied in order to 
produce a better safety plan for marine vessels and offshore structures. Advance development in computational instrument since the early 2000 s is introducing a numerical method as an alternative virtual experiment. Its characteristic as a virtual simulation provides practicality in conducting large-scale analysis.

Finite element (FE) approach is one of these methods, and it was judged well enough to model nonlinear phenomena, such as ship collision [9-10], and grounding [11-12] by researchers in impact analysis for maritime transportation. A nonlinear fashion is preferred to be used in impact analysis. Even though nonlinear analysis is a more complex approach, but it results in a more accurate solution than linear analysis when the basic assumptions of linear analysis are violated. Nonlinear FE is a match for several calculations, such as a structure/infrastructure with dynamic (time-dependent) loads, large deformations, and nonlinear materials such as metals, beyond its yield point [13]. Impact phenomena, such as collision and grounding, have small time processes, explicit methodology in FE approach is matched with this criterion. Using this methodology to solve the nonlinear problem, a central difference time integration method is used, and accelerations evaluated at time $t$ are given in Equations 1 to 5 [14].

$$
\begin{gathered}
\left\{a_{t}\right\}=[M]^{-1}\left(\left\{F_{t}^{\text {ext }}\right\}-\left\{F_{t}^{\text {int }}\right\}\right) \\
F_{t}^{\text {int }}=\sum\left(\int_{\Omega}\left(B^{T} \sigma_{n} d \Omega+F^{h g}\right)+F^{\text {contact }}\right) \\
\left\{v_{t+\Delta t / 2}\right\}=\left\{v_{t-\Delta t / 2}\right\}+\left\{a_{t}\right\} \Delta t_{t} \\
\left\{u_{t+\Delta t}\right\}=\left\{u_{t}\right\}+\left\{v_{t+\Delta t / 2}\right\}+\left\{a_{t}\right\} \Delta t_{t+\Delta t / 2} \\
\left\{x_{t+\Delta t}\right\}=\left\{x_{0}\right\}+\left\{u_{t+\Delta t}\right\}
\end{gathered}
$$

where $a_{t}=$ the acceleration at time $t ; v_{t}=$ the velocity at time $t ; u_{t}=$ the displaced distance at time $t ; M=$ the lumped mass matrix; $x_{0}=$ the initial geometry position; $x_{t}=$ the updated geometry position at time $t ; F_{t}^{\text {ext }}=$ the applied external and body force vector; $F_{t}^{\text {int }}=$ the internal force vector (major computational expense); $F^{\text {hg }}=$ the hourglass force; and $F^{\text {contact }}=$ the contact force.

\section{Research method and preparation}

\subsection{Ship model}

The target ship is numerically modelled after a $144 \mathrm{~m}$ non-polar class chemical tanker, and the deformable characteristic is implemented on the ship geometry. Modelling is focused on the double bottom structure part in order to reduce simulation time. The numerical model of the ship geometry is shown in Fig. 1. Shell element formulation considers a fully integrated version of the Belytschko-Tsay to be applied to the structure in order to avoid hourglass and shear-locking phenomena. Ship material is embedded by high-strength low-alloy steel with density $\rho=7850 \mathrm{~kg} . \mathrm{m}^{-3}$; Poisson's ratio $v=0.29$; Cowper-Symonds parameter $C=3200 \mathrm{~s}^{-}$ ${ }^{1}$; Cowper-Symonds parameter $P=5$; and yield strength $\sigma_{Y}=350 \mathrm{MPa}$. 


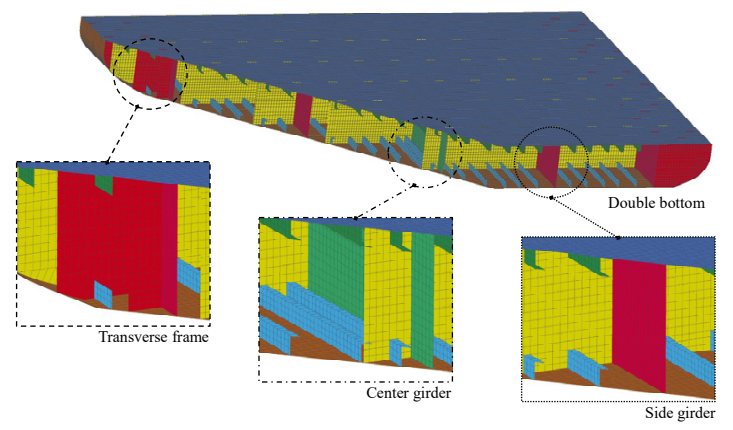

Fig. 1. Structural model of the double bottom. Inner components are highlighted by the circles.

\subsection{Grounding scenario}

During grounding analysis, the boundary condition (Fig. 2) is necessary to be applied. The ship is set to be fixed on the centerline and the end of the bottom model is restrained. The indenter approaches with an applied velocity of $10 \mathrm{~m} / \mathrm{s}$ into three locations, namely center and side girders for longitudinal component and the space between two girders. The coefficient of the entities' friction and the automatic surface-to-surface contact are chosen in the contact configuration between ship and obstruction. The defined model and impact configuration are calculated by finite element (FE) codes ANSYS LS-DYNA [14] to produce structural estimation during ship grounding.

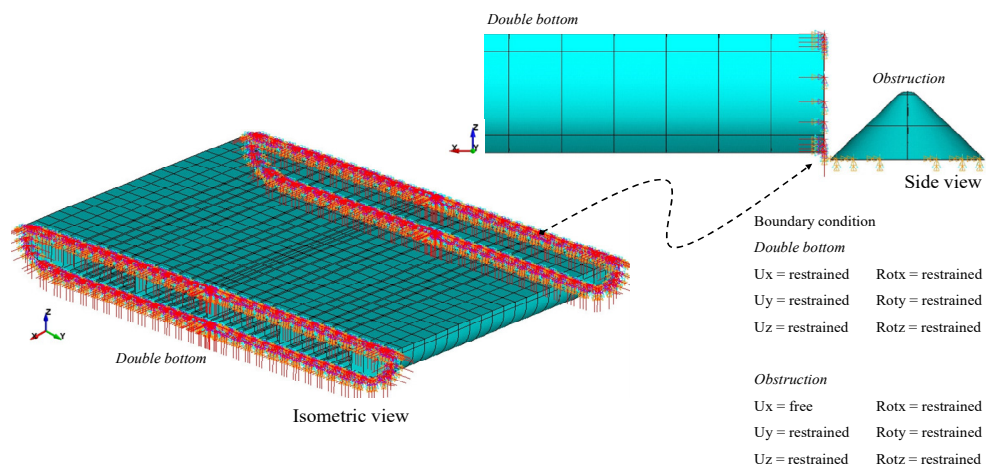

Fig. 2. Brief illustration of the boundary conditions on the ship and obstruction in grounding.

The underwater seabed is defined as a conical rock to be the obstruction in the first case, where the characteristic of the plagioclase mineral is augmented into the conical rock [1516]. Comparison of the material's contribution to the structural crashworthiness is conducted with the deployment of a solid-ice model [17] in the second case. The obstruction is assumed to be entirely rigid in order to observe the deformation pattern and damage extent only on the double bottom structure. The Coulomb friction coefficient 0.3 is applied for rock mineral; steel interaction on the first case and ice-steel coefficients based on a laboratory experiment by Cho et al. [18] with values $\mu_{s}=0.05$ and $\mu_{k}=0.03$ are used for the second case. Failure of the bottom plate and other components is expected after they surpass the ultimate strain during contact with the rock. Therefore, it is necessary to define strain value as a function of structural failure which criterion of Germanischer Lloyd [19] applied to the ship. 


\section{Results and discussion}

Structural behaviours under ship grounding to several targets are evaluated. Crashworthiness criteria are discussed in this section. The first criterion is the resultant force which is presented in Fig. 3a. It can be observed that the impact of indenter and target ship took place after $0.02 \mathrm{~s}$. At this moment, the remarkable fluctuation occurred as resistance capability of the bottom structure's components on the initial impact was very strong. The structure in the location of the initial contact consisted T-intersection which is formed of the bottom girder and transverse floor.

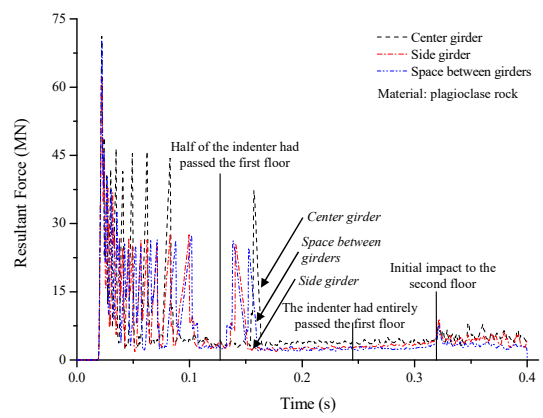

(a)

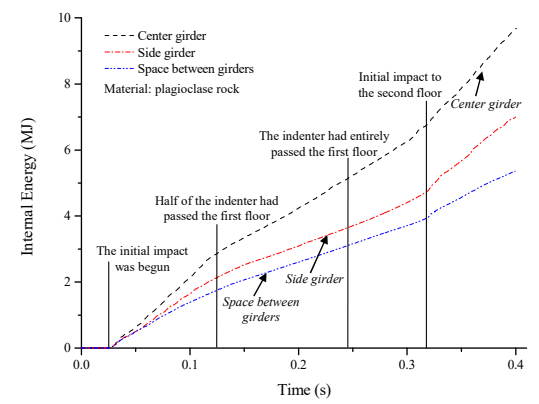

(b)

Fig. 3. Responses under rock grounding: (a) resultant force and (b) internal energy.

The intersection was also strengthened by its connection to the bottom shell. The high fluctuation occurred for the first two grounding models as longitudinal girders were the target components in these scenarios. In another scenario where the target was determined to be the space between two girders, the force magnitude was to be found smaller. It is evidence that the space was the weakest part among all determined scenarios. It can be denoted as the critical location, since larger rocks may cause higher damage extent that can be produced after impact to this component, and a remarkable oil spill after grounding.

An observation in terms of structural resistance can be more comfortable to be performed on the internal energy as the second criterion. This energy represents how hard it is for a structure to be plastically deformed or even crushed during rock penetration. It is mostly used for evaluation on the deformable body during impact between two entities. In the discussion, this criterion describes the energy magnitude (Fig. 3b) that was experienced by each target. After initial impact, similarity was produced for three targets. As penetration of the rock on the center girder advances, the energy increment indicated that remarkable resistance occurred before half of the rock completely penetrated the first transverse floor.

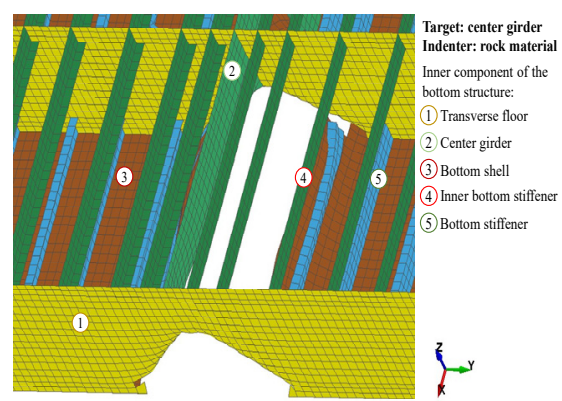

(a)

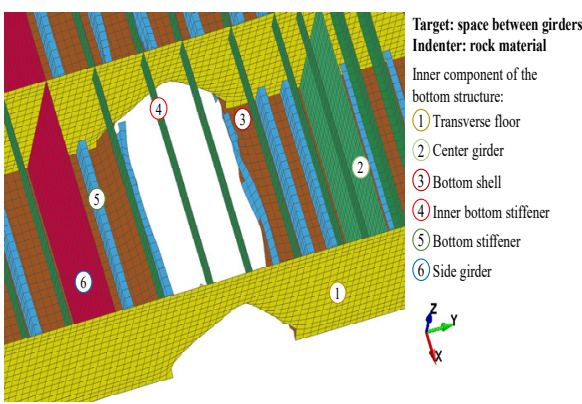

(b)

Fig. 4. Damage on the bottom structure after grounding: (a) bottom (longitudinal) girder and (b) space between side-center girders. 
After passing this point, the energy for all locations increased significantly. Confirmation of prior observation on the resultant force was confirmed after evaluating the internal energy, where the space between two girders (center and side girders) produced the lowest energy. It means that it needs a smaller energy than the other two locations to crush or penetrate this target in hard grounding scenario. The deformation pattern between two scenarios, i.e. impacts to girder and space, is presented in Fig. 4. It is obtained from the illustration that the lower part of the girder was severely damaged during penetration and the deformation pattern on the bottom plate matched with the description of clean curling cut. In the grounding to the space between girders, only the longitudinal stiffener of the bottom shell strengthened the structure. This structural arrangement makes a less destroyed component that is located on the space which leads to less internal energy. Satisfactory calculation results is successfully achieved as the tendency fulfilled the damage-energy relation (see the empirical formula and its application in Bae et al. [13].

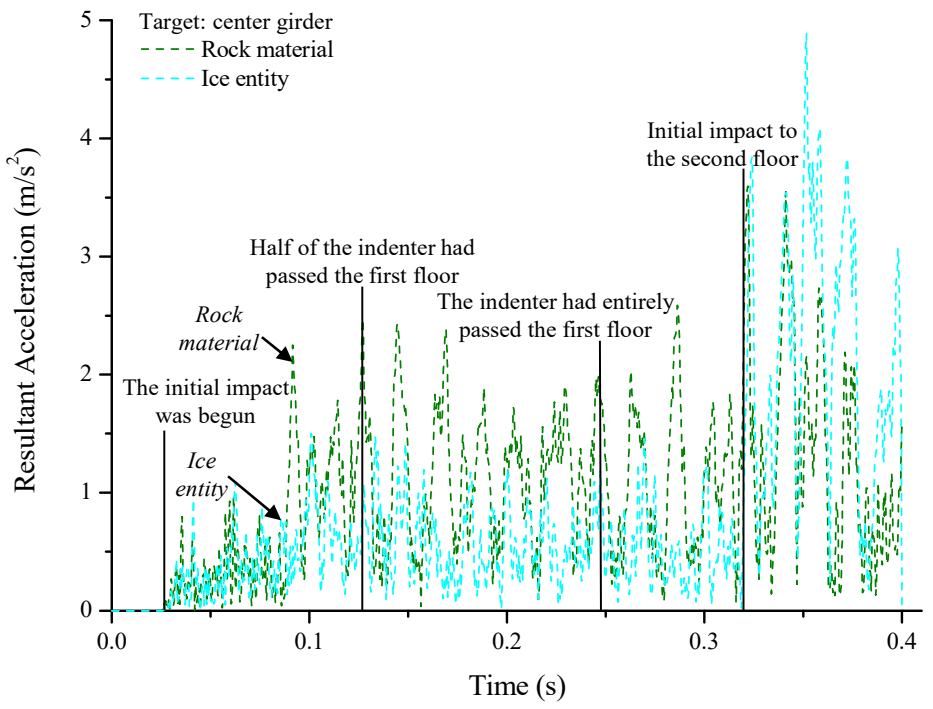

Fig. 5. Crashworthiness criterion - structural acceleration during impact with different obstructions.

Besides comparison between target locations, the contribution of the obstruction material to the structural behavior will be discussed. The third criterion is the resultant acceleration of the bottom structure. It is shown in Fig. 5 that the rock material which was harder than the ice entity produced higher fluctuation during the grounding process. This result also indicated that even though the obstruction was entirely modelled as a rigid body, contribution of material properties could not be exempted. The nature of the material type, such as hardness and friction, will provide a direct contribution to structural crashworthiness during impact (such as collision and grounding). Based on material properties and their comparison in Table 1, it was concluded that Poisson's ratio $\mathrm{v}$ and Young's modulus $E$ were the most influencing parameters to the obstructions' hardness. Modelling of the indenter using rigid body is very useful for two assumptions: first, it can assess structural deformation and response in a critical scenario in which the obstruction's shape is entirely constant during penetration. This situation will produce larger damage than an obstruction that can be deformed during contact with another object. Second, it can reduce time simulation in which deformation contour and other structural responses (stress, strain, deformation etc.) of the obstruction is neglected during calculation. It can be very helpful for analysis of various nonlinear interactions with a limited-performance computational instrument. 
Table 1. Material properties of the obstructions and comparison between the obstruction's properties.

\begin{tabular}{|c|c|c|c|c|}
\hline \multirow{2}{*}{ Properties } & $\boldsymbol{\rho}$ & $\boldsymbol{\mu}$ & $\boldsymbol{v}$ & $\boldsymbol{E}$ \\
\cline { 2 - 5 } & $\mathbf{( k g . m}^{\mathbf{3}} \mathbf{)}$ & $\mathbf{( - )}$ & $\mathbf{( - )}$ & $\mathbf{( M P a )}$ \\
\hline Plagioclase & 2690 & 0.3 & 0.296 & 67450 \\
\hline Ice & 870 & $0.03-0.05$ & 0.003 & 9000 \\
\hline Difference (approximation) & $68 \%$ & $83 \%$ & $99 \%$ & $87 \%$ \\
\hline
\end{tabular}

A verification of the resultant acceleration is presented by the kinetic energy (Fig. 6) as the final criterion. The ice model which has smaller density will be lighter than the rock. This correlation is given in the mass-density-volume relation $(\rho=M / V)$ which for the same volume, the higher density will give bigger mass to an object. The ice entity which was lighter than the oceanic rock produced smaller kinetic energy during penetration on the bottom structure. On the other hand, the kinetic energy of the rock material was significantly higher for the entire grounding process. The tendency of both materials was observed to be similar due to the influence of constant velocity on the obstruction, and the same target component on the double bottom structures.

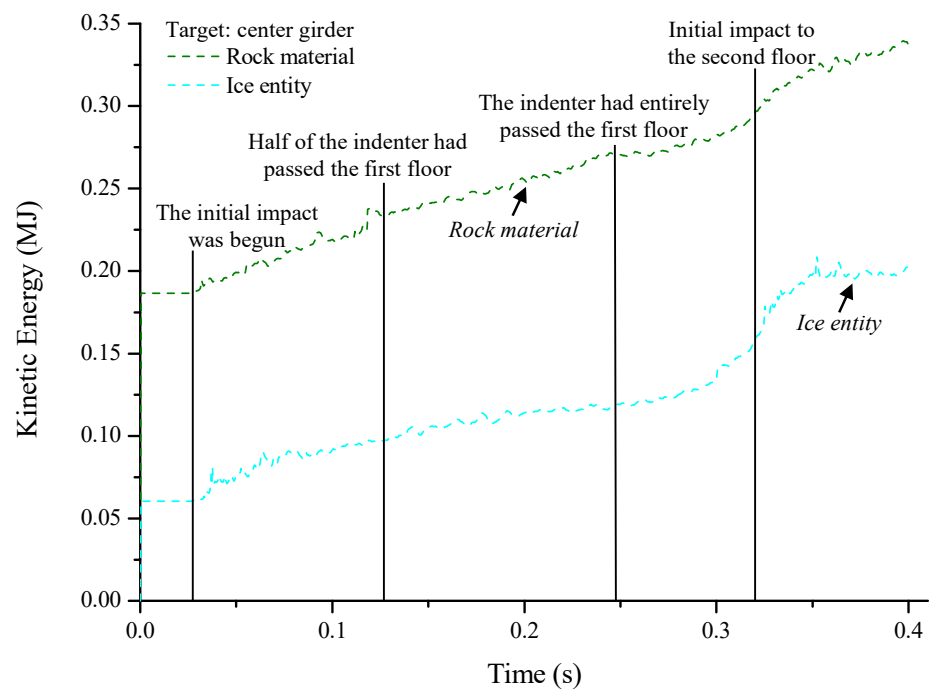

Fig. 6. Kinetic energy in uniform velocity during impact with different obstructions.

Increment for the ice obstruction after the initial impact to the second transverse floor was influenced by stacked structural components in the penetration process. Dissimilar to the plagioclase which has higher friction and mass, lighter ice material could not make the deformed component surpass their strain limit and get them erased in the analysis. These components were piled-up in the ice grounding which affected the increment of the kinetic energy as the stacked components were harder to penetrate.

\section{Conclusion}

This work presented a series of grounding scenarios which considered target location and material type of the obstruction as the influenced parameters to crashworthiness criteria. The results were concluded to be correlated to each other as confirmation of the resultant force was given by the internal energy, and contribution of material properties in impact 
was presented by the resultant acceleration and confirmed by the kinetic energy. It was evidenced based on calculation results that in terms of the material type, the definition of the obstruction as a rigid object was presented satisfactorily on structural criteria and failure sequences. Observation on the structural damage indicated that for the composed head-on scenarios, damage mainly occurred on the bottom shell and on other components that were connected to it. Status of the inner bottom shell which was considered vital for cargo safety was confirmed to be in a good condition after groundings. It is recommended to conduct an observation of the crushing of ice obstruction, and the effect of individual material properties (e.g. $E$, $v$, etc.) to structural responses.

\section{References}

1. Allianz, Safety and shipping 1912-2012, from Titanic to Costa Concordia (Allianz Global Corporate and Specialty, Munich, 2012)

2. A.R. Prabowo, D.M. Bae, J.H. Cho, J.M. Sohn, Lat. Am. J. Sol. Struct. 14, 1594 (2017)

3. A.R. Prabowo, S.J. Baek, H.J. Cho, J.H. Byeon, D.M. Bae, J.M. Sohn, Lat. Am. J. Sol. Struct. 14, 1345 (2017)

4. A.R. Prabowo, D.M. Bae, J.M. Sohn, A.F. Zakki, B. Cao, J.H. Cho, Thin-Walled Struct. 115, 225 (2017)

5. D.M. Bae, A.R. Prabowo, B. Cao, J.M. Sohn, A.F. Zakki, Q. Wang, Lat. Am. J. Sol. Struct. 13, 2991 (2016)

6. B.C. Simonsen, Mechanics of ship grounding (Technical University of Denmark, Lyngby, 1997)

7. S. Otto, P.T. Pedersen, M. Samuelides, P.C. Sames, Mar. Struct. 15, 461(2002)

8. H. Zhiqiang, J. Amdahl, H. Lin, Mar. Struct. 24, 436 (2011)

9. A.R. Prabowo, J.M. Sohn, D.M. Bae, J.H. Cho, Curved and Layer. Struct. 4, 225 (2017)

10. A.R. Prabowo, D.M. Bae, J.M. Sohn, A.F. Zakki, B. Cao, Open Eng. 7, 91 (2017)

11. O.V.E. Sormunen, M. Kõrgesaar, K. Tabri, M. Heinvee, A. Urbel, P. Kujala, Mar. Struct. 50, 205 (2016)

12. A. AbuBakar, R.S. Dow, Int. J. Sol. Struct. 50, 623 (2013)

13. D.M. Bae, A.R. Prabowo, B. Cao, A.F. Zakki, G.D. Haryadi, J. Mar. Sci. App. 15, 63 (2016)

14. ANSYS, ANSYS LS-DYNA User's Guide (ANSYS. Inc, Pennsylvania, 2017)

15. N.I. Christensen, J. Geophys. Res. 101, 3139 (1996)

16. A.R. Prabowo, B. Cao, D.M. Bae, S.Y. Bae, A.F. Zakki, J.M. Sohn, Lat. Am. J. Sol. Struct. 14, 1 (2017)

17. R.E. Gagnon, J. Wang, Cold Reg. Sci. Tech. 81, 26 (2012)

18. S.R. Cho, E.J. Chun, C.S. Yoo, S.Y. Jeong, C.J. Lee, J. Soc. Nav. Arch. Korea 48, 363 (2011) - in Korean.

19. Germanischer Lloyd, Development of explanatory notes for harmonized SOLAS Chapter II-1 (International Maritime Organization, London, 2003) 